\title{
Fibromatosis Colli: A Review of 4 Cases
}

\author{
Maha Hakimi, Omar Oulghoul, Benhoummad Othmane, Youssef Rochdi, and Abdelaziz Raji
}

\section{ABSTRACT}

Fibromatosis colli is a rare congenital pseudotumor of the sternocleidomastoid muscle, mainly found in the pediatric population. We propose to present four cases diagnosed in the ENT-Neck and head surgery department of the university medical center Mohammed VI in Marrakech, moreover we will recap the management strategy of this entity but also its evolution. We received in the pediatric emergency three infants of 1,3 and 6 months old and one child of 7 years old, with as a main symptom a painful congenital torticollis. The ultrasound added to our clinical inspection allowed us fairly easily to diagnose the patients. No drug or surgical treatment was prescribed in the infant cases, though surgical treatment was inevitable in the 7 years old patient's case. During the follow-up, carried out each month, we observed a gradual regression of swelling and torticollis. The prevalence of $\mathrm{FC}$ is estimated at $0.3-2 \%$ of births. The diagnosis is made at the age of 3 to 4 weeks of life by a thorough inspection of a torticollis associated with a later cervical mass in the framework of a difficult delivery (use of forceps or suction cup). Ultrasonography allows us to diagnose this entity due to the typical aspect of a fusiform thickening of the Sternocleidomastoid muscle. Surgical and medical treatment is rarely useful, and only symptomatic treatment is indicated. The spontaneous evolution of Fibromatosis colli is, even in the absence of treatment, the regression spontaneously in 4 to 6 months. Fibromatosis colli is a relatively rare entity, its frequency in Africa remains to be established, but it is clear that an early diagnosis is key to a speedy recovery.

Keywords: Congenital Torticollis, Fibromatosis coli, Physiotherapy.
Submitted : April 21, 2021

Published : May 13, 2021

ISSN: $2593-8339$

DOI: $10.24018 /$ ejmed.2021.3.3.827

\section{Maha Hakimi*}

ENT - Neck and Head surgery Department, University Medical Center Mohammed VI, Marrakech, Morocco.

(e-mail: mahahakimi1@gmail.com)

Omar Oulghoul

ENT - Neck and Head surgery Department, University Medical Center Mohammed VI, Marrakech, Morocco.

(e-mail: oulghoul@gmail.com)

Benhoummad Othmane

ENT - Neck and Head surgery Department, University Medical Center Mohammed VI, Marrakech, Morocco.

(e-mail: medoth1@hotmail.com)

Youssef Rochdi

ENT - Neck and Head surgery Department, University Medical Center Mohammed VI, Marrakech, Morocco.

(e-mail: rochdi_86@ ${ }^{\circledR}$ hotmail.com)

Abdelaziz Raji

ENT - Neck and Head surgery Department, University Medical Center Mohammed VI, Marrakech, Morocco.

(e-mail: raji.abdelaziz ${ }^{@}$ gmail.com)

*Corresponding Author

\section{INTRODUCTION}

Fibromatosis colli is a rare congenital pseudotumor of the sternocleidomastoid muscle, found mainly in the pediatric population [1]. Although its etiology is still unknown to this day, its main symptom remains a congenital torticollis and ultrasound is usually sufficient for diagnosis, although in some cases MRI and CT are required. The treatment of this relatively rare pathology is conservative and allows a spectacular evolution in 4 to 6 months. We propose to present 4 diagnosed cases in the ENT-Head and neck surgery department of Mohammed VI medical center of Marrakech, we will also summarize the management of this entity and its evolution.

\section{CASES}

We received in the pediatric emergencies of Mohammed VI medical center three infants of 1, 3 and 6 months old and one child of 7 years old, with as a main symptom in all cases a painful congenital torticollis which is both functionally and esthetically handicapping (Fig. 1 and 2).

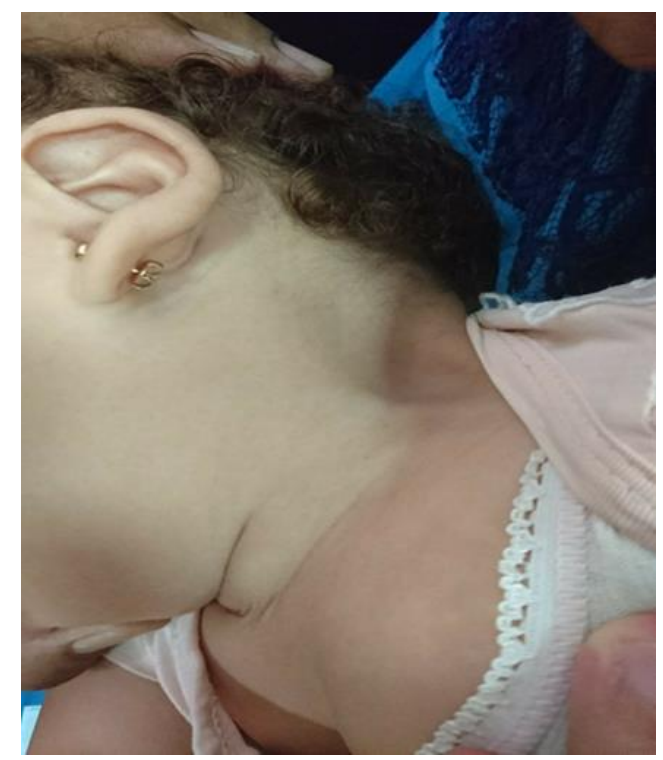

Fig. 1. Congenital swelling in a 3-month-old infant. 


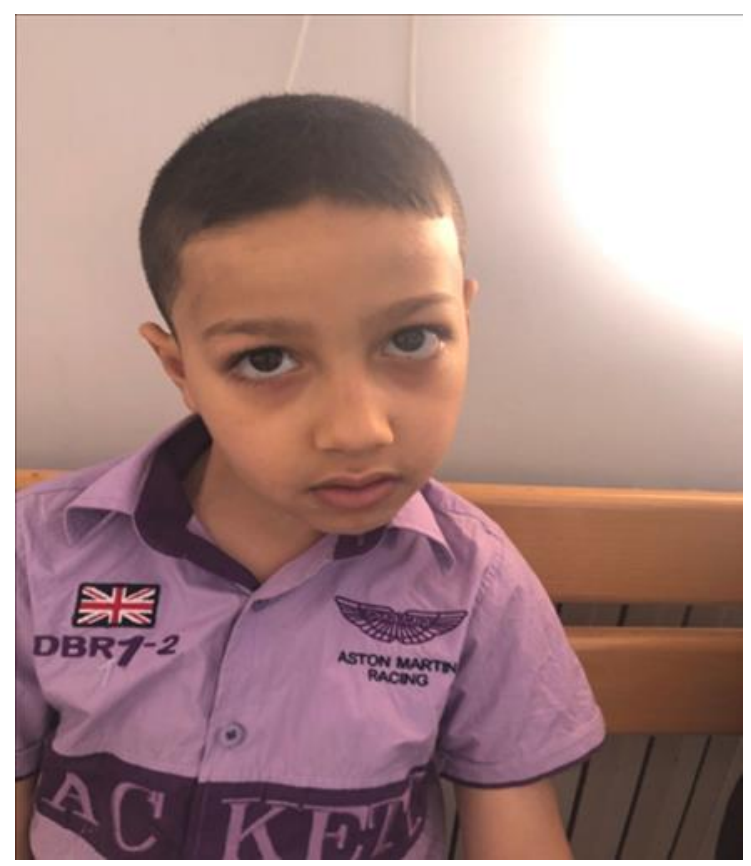

Fig. 2. 7-year-old child with fibromatosis colli.

All children but one was male. All born to a term pregnancy by cephalic presentation, there was also one case of primiparous mother, all the others were multiparous, plus all the pregnancies did not have any specific incident. The birth weight of all patients was normal with a correct weightheight evolution for their ages. There was no pathological family history and no inbreeding link between the parents. While collecting the history data of the patients, the neck swelling in all cases was noted already at 1 month old, the attention of the mother having been attracted in all cases by a permanent deviation of the head on the right side. There was no notion of fever or trauma. Ultrasonography, performed in all cases as a first step, noted a fusiform thickening of the body of the Sternocleidomastoid muscle with retention in all cases of the fibrillar aspect of the muscle. The rest of the exam was normal. CT and MRI were not requested in any of the cases. Given the ultrasound results and the clinical aspect, the diagnosis of Fibromatosis Colli was retained. No medical treatment was prescribed in any case. Infants were rehabilitated, and the mother was advised to carry the baby as often as possible to the back, with the face facing the side of the thickening. During monthly follow-ups, we observed a progressive regression of swelling and torticollis. Only one case was surgically treated with partial Sternocleidomastoid muscle section and patient's neck immobilization and concomitant rehabilitation, it was the 7-year-old case, after we noted no improvement and an important functional handicap to the child (Fig. 3). The evolution over 6 months, in all cases including the surgical one, noted a disappearance of torticollis and laterocervical mass.

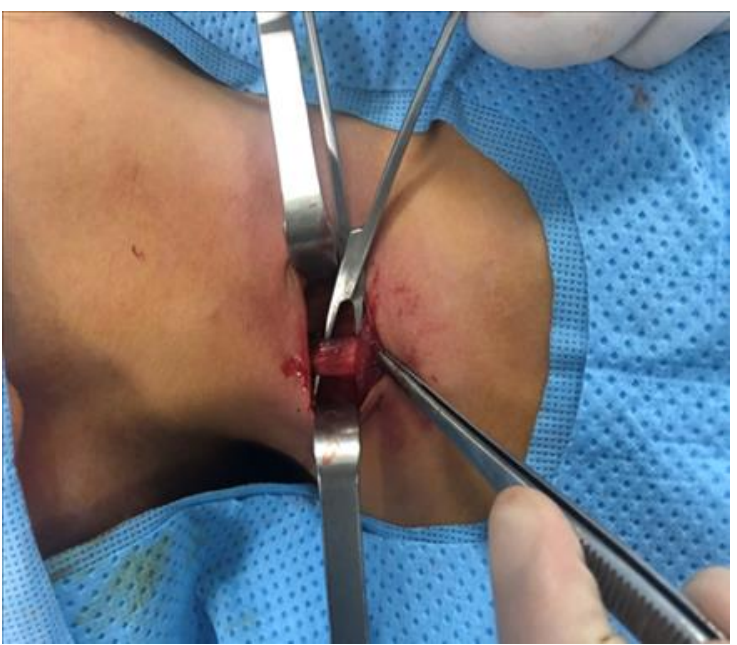

Fig. 3. Thickening of SCM muscle as shown during the surgical procedure.

\section{DISCUSSION}

The prevalence of Fibromatosis Colli is estimated at 0.3$2 \%$ of births, making it a relatively rare disease, with a clear male predominance [2]. Nevertheless, its prevalence remains unknown in Africa, except a study by Abdur-Rahman LO and al of 15 cases collected in 10 years (1999-2008) in University Hospital in Nigeria [3]. Usually, the diagnosis is made at the age of 3 to 4 weeks of life, as it should be, by the family of the infant due to a torticollis associated with a laterocervical mass in the context of a difficult delivery (use of forceps or suction pad). However, in African countries, such as Morocco, there is a diagnostic delay, as is the case of our patients diagnosed at 7 years old for the oldest. Indeed, many parents seek counsel pretty late because they assume that the abnormal position of the head of the child is due to the nonacquisition of holding of the head which is normally acquired only at 3 months old in the normal psychomotor development of the child. Its etiopathogenesis is unclear to this day and many theories coexist. The most common is an abnormality of Sternocleidomastoid muscle due to fibrosis and muscle contracture secondary to a syndrome of the lodges and ischemic lesions favored by intra uterine fetal malposition [4]. Another theory would be rather in favor of trauma or microtrauma of the Sternocleidomastoid muscle during laborious childbirth [5]. Most likely, these two theories complement each other. Ultrasonography has allowed us, as for several authors, to diagnose, fairly easily, this entity. The typical aspect is a fusiform thickening of the Sternocleidomastoid muscle, there is also no vascular and lymphatic involvement. It is, by far, the instrument of choice to identify Fibromatosis Colli. Some authors believe that the clinical examination is sufficient for the diagnosis and that no further investigation should be carried out routinely, but they nevertheless recognize the usefulness of ultrasound to eliminate other diagnoses when the clinical aspect is atypical [5]. The differential is mainly with rhabdomyosarcoma and neuroblastoma, in which in addition to a cervical mass, there are lymphadenopathies, vascular embedding or invasion of adjacent structures. Surgical and medical treatment is rarely useful, and only symptomatic treatment is indicated including physiotherapy or warm-up exercises. Surgical treatment is indicated in cases diagnosed after the age of 6 months or in cases resistant to symptomatic treatment. The spontaneous 
evolution of Fibromatosis Colli can be, even in the absence of treatment, the spontaneous regression of the mass in 4 to 6 months [6].

\section{CONCLUSION}

Fibromatosis colli is a relatively rare entity, its frequency in Africa remains to be established. Its diagnosis combines clinical and ultrasound examination, which eliminates other causes of torticollis in newborns and infants. Regression under physiotherapy is the norm and is spectacular in 4 to 6 months.

\section{ACKNOWLEDGMENT}

The authors declare no conflict of interest.

Oral consent of the patients was obtained for the publication of these cases.

\section{REFERENCES}

[1] S. Smiti, Naveen M. Kulkarni, and Jyoti Singh. Case Report: Fibromatosis colli in a neonate Indian J Radiol Imaging. 2010 Feb; 20(1): 45-46.

[2] Garetier M, Breton S, Pennaneach A, Barberot C, Chinellato S, Rousset J. Fibromatosis colli. Presse Medicale. 2012;41(2):213-214.

[3] AbdurRahman LO, Cameron BH. Sternomastoid tumor of infancy and congenital muscular torticollis. In: Ameh EA, Bickler SW, Lahoo K, Nwomeh BC, Poenaru D, editors. Pediatric Surgery: a comprehensive text for Africa. Global HELP. 2010. pp. 448-453.

[4] Kumar B, Pradhan A. Diagnosis of sternomastoid tumor of infancy by fine-needle aspiration cytology. DiagnCytopathol. 2011 Jan;39(1):137.

[5] Adamoli P, Longo P, Falsaperla R, et al. Rapid spontaneous resolution of fibromatosis colli in a 3-week-old girl. Case Rep Otolaryngol. 2014; 2014:264940.

[6] Cheng JC, Wong MW, Tang SP, Chen TMK, Shum SLF, Wong EMC. Clinical determinants of the outcome of manual stretching in the treatment of congenital muscular torticollis in infants A prospective study of 821 cases. J Bone Joint Surg Am. 2001 May;83-A (5):679-87. 